\title{
Thermonuclear supernova simulations with stochastic ignition
}

\author{
W. Schmidt and J. C. Niemeyer
}

Lehrstuhl für Astronomie, Institut für Theoretische Physik und Astrophysik, Universität Würzburg, Am Hubland, 97074 Würzburg, Germany

e-mail: schmidt@astro.uni-wuerzburg.de

Received 15 July 2005 / Accepted 11 October 2005

\section{ABSTRACT}

We apply an ad hoc model for dynamical ignition in three-dimensional numerical simulations of thermonuclear supernovae assuming pure deflagrations. The model makes use of the statistical description of temperature fluctuations in the pre-supernova core proposed by Wunsch \& Woosley (2004, ApJ, 616, 1102). Randomness in time is implemented by means of a Poisson process. We are able to vary the explosion energy and nucleosynthesis depending on the free parameter of the model which controls the rapidity of the ignition process. However, beyond a certain threshold, the strength of the explosion saturates and the outcome appears to be robust with respect to the number of ignitions. In the most energetic explosions, we find about $0.75 M_{\odot}$ of iron group elements. Other than in simulations with simultaneous multi-spot ignition, the amount of unburned carbon and oxygen at radial velocities of a few $10^{3} \mathrm{~km} \mathrm{~s}^{-1}$ tends to be reduced for an ever increasing number of ignition events and, accordingly, more pronounced layering results.

Key words. stars: supernovae: general - methods: numerical - stars: evolution - stars: interiors

\section{Introduction}

The significance of the ignition characteristics at the onset of thermonuclear supernova explosions has been recognised since the advent of multi-dimensional numerical simulations. The mounting evidence for diversity among observed type Ia supernovae renders the quest for realistic modelling of the ignition process yet more topical (Benetti et al. 2005; Leonard et al. 2005; Stritzinger et al. 2005). Substantial efforts have been made to analyse the effect of choosing different initial burning regions in simulations of thermonuclear supernovae (García-Senz \& Bravo 2005; Röpke \& Hillebrandt 2005). The multi-point ignition scenarios which have recently been presented by Röpke et al. (2005) suggest that the energy generation becomes maximal for about one hundred ignitions. In this case, unburned carbon and oxygen at low radial velocity largely diminish. Except for the two-dimensional study by Livne et al. (2005), however, it has not been attempted so far to generate ignitions at different instants of time during a simulation.

In this article, we present a simple stochastic model for the dynamical ignition of thermonuclear deflagration in threedimensional simulations. The purpose of the model is an investigation of the possible consequences of using a simple parametrisation for the ignition probability. In particular, the total amount of iron group and intermediate mass elements as well as their spatial distribution and density functions in radial velocity space are studied. We do not claim yet that the highly complex physics of the ignition process in thermonuclear supernova is fully taken into account. Notwithstanding the tentative nature of the stochastic ignition model, it may help to clarify the extent of possible variations in the yields and the distribution of burning products without invoking a hypothetical deflagration to detonation transition (Gamezo et al. 2004, 2005). Whether the predictions of the model can be reconciled with properties of observed type Ia supernovae ( $\mathrm{SNe}$ Ia) will depend on further developments in the theoretical understanding of convection and the birth of flames in the pre-supernova core.

\section{Stochastic ignition}

The simplest thermodynamical description of the presupernova core is an adiabatic background plus temperature fluctuations due to convection driven by gradual thermonuclear burning. For the background temperature, we assume the parabolic profile

$T_{\mathrm{a}}(r)=\max \left\{T_{0}\left[1-\left(\frac{r}{\Lambda}\right)^{2}\right], T_{\text {ext }}\right\}$

with a cutoff at the thermal radius $\Lambda=7.35 \times 10^{7} \mathrm{~cm}$ for the central density $\rho_{\mathrm{c}}=2 \times 10^{9} \mathrm{~g} \mathrm{~cm}^{-3}$ (Wunsch \& Woosley 2004) and an isothermal exterior region with $T_{\text {ext }}=5 \times 10^{5} \mathrm{~K}$. For the temperature fluctuations, Wunsch \& Woosley (2004) proposed a statistical model on the basis of the mixing-length approach. The typical temperature excess $\delta T_{\mathrm{b}}$ gained by fluid elements relative to the adiabatic background in a spherically symmetric core region with Kolmogorov power spectrum is given by

$\frac{\delta T_{\mathrm{b}}}{T_{0}} \sim\left(\frac{\Lambda}{R}\right)^{4 / 7}\left(G \rho_{0} \delta_{\mathrm{P}} \tau^{2}\right)^{-3 / 7}$ 
where $R$ can be interpreted as mixing length, $\delta_{\mathrm{P}}$ is the logarithmic derivative of density relative to pressure and $\tau$ is the nuclear time scale (see Wunsch \& Woosley 2004, Eqs. (9) and (18)).

The ignition algorithm utilised in our simulations is based on a discrete stochastic process, namely, the Poisson process. The probability for $i$ ignitions within the time interval $\delta t$ is given by

$P_{t, r}(i, \delta t)=\frac{\left(v_{t, r} \delta t\right)^{i}}{i !} \exp \left(-v_{t, r} \delta t\right)$

where $v_{t, r}$ is a function of the temperature excess $\delta T_{\mathrm{b}}$ and the local thermodynamic background state. Assuming that the ignition events are statistically independent, the number of ignitions per unit time increases proportional to the volume of fuel within a region of approximately constant background conditions. For this reason, we consider the ignition probability $P_{t, r, \delta r}(i, \delta t)$ for the unburned material within spherical shells of width $\delta r \ll R$. On grounds of ergodicity, the increase of probability in proportion to the volume is equivalent to increasing either the sampling time $\delta t$ or the parameter $v_{t, r}$ by the ratio of the fuel volume relative to a unit volume. Thus, $P_{t, r, \delta r}(i, \delta t)$ is given by an expression analogous to the right hand side in Eq. (3) with $v_{t, r}$ replaced by

$v_{t, r, \delta r}=\left\langle v_{t, r} \theta[G(t, \boldsymbol{r})]\right\rangle_{r, \delta r} \frac{3 r^{2} \delta r}{r_{\mathrm{b}}^{3}}$,

where $G(t, \boldsymbol{r})$ is the level set function which represents the flame front, $\theta[G(t, \boldsymbol{r})]$ is zero for $G(t, \boldsymbol{r})<0$ (i.e. in ash regions) and unity for $G(t, \boldsymbol{r})>0 .\langle\rangle_{r, \delta r}$ denotes the average over a spherical shell of radius $r$ and width $\delta r$. For the normalisation, we choose a sphere of radius $r_{\mathrm{b}}$ which is roughly the size of the central heating region (Wunsch \& Woosley 2004, Eq. (24)). The underlying assumption of spherical symmetry is, of course, increasingly flawed in the course of the explosion. However, we found empirically that the spatial variation of the background state variables within the unburned material contained in a thin spherical shell at each given instant of time is small.

In order to complete the model, we formulate a hypothesis for $\left\langle v_{t, r} \theta[G(t, \boldsymbol{r})]\right\rangle_{r, \delta r}$. Since $v_{t, r}$ is an inverse time scale which determines the probability of ignition per unit time, we conjecture that

$\chi_{\mathrm{u}, r, \delta r}(t)^{-1}\left\langle v_{t, r} \theta[G(t, \boldsymbol{r})]\right\rangle_{r, \delta r}=C_{\mathrm{e}} \frac{S_{\mathrm{nuc}}}{C_{\mathrm{P}} \delta T_{\mathrm{b}}}$,

where $c_{\mathrm{P}}$ is the heat capacity and $S_{\text {nuc }}$ is the nuclear energy generation rate for the average temperature and mass density, respectively, of unburned material within a particular spherical shell (Woosley et al. 2004, Eqs. (4) and (6)). The exponentiation parameter $C_{\mathrm{e}}$ determines the overall rapidity of the ignition process. The filling factor $\chi_{\mathrm{u}, r, \delta r}(t)$ specifies the volume fraction of unburned fluid at time $t$ within the shell. Note that both sides of Eq. (5) are independent of the amount of fuel which is consistent with the statistical nature of the quantities involved.

\section{Numerical simulations}

For the numerical simulations presented in the following, we use the same techniques as described in Röpke \& Hillebrandt (2005), except for a hybrid grid geometry with a central uniform part and exponentially increasing grid size outside (Röpke et al. 2005) in combination with a localised subgrid scale model which is introduced in Schmidt et al. (2005). Owing to the co-moving hybrid grid, we were able to achieve sensible results with merely $128^{3}$ numerical cells in one octant. The subgrid scale model is based on the hydrodynamical equation for the turbulence energy which was adopted by Niemeyer \& Hillebrandt (1995) for the numerical simulation of thermonuclear supernovae. In the new implementation, we apply dynamical procedures for the in situ calculation of closure parameters. Moreover, an additional term was added to the subgrid scale turbulence energy equation which accounts for buoyancy effects on unresolved scales (Schmidt et al. 2005). Thereby, the Sharp-Wheeler relation for the characteristic velocity scale of the Rayleigh-Taylor instability is effectively incorporated into the turbulence energy model. In addition, the localised SGS model features an algorithm which corrects the SGS turbulence energy for the effect of the shifting cutoff length scale of the co-moving grid, because the gradually increasing cutoff length entails an intrinsic growth of SGS turbulence energy (Schmidt et al. 2005).

The numerical implementation of the Poisson process was adopted from Press et al. (1996). For each ignition event in a particular Poisson process realisation, a random point is sampled from a uniform spatial distribution and projected onto the spherical shell associated with the process. If the resulting point happens to fall inside fuel, a small bubble of burned material with radius $1.5 \Delta$, where $\Delta$ is the numerical cell size, is inserted. This algorithm implicitly accounts for the filling factor $\chi_{\mathrm{u}, r, \delta r}(t)$ of the fuel in each shell. For the width of the spherical shells we chose $\delta r=2 \Delta$.

We performed simulations with different values of $C_{\mathrm{e}}$ both in octants and full spherical domains. The influence of different mixing lengths $R$ relative to the thermal radius $\Lambda$ was investigated as well. The simulation parameters as well as the total number of ignitions, the explosion energetics and the burning product masses are listed in Table 1 . In one series of simulations the value of the exponentiation parameter $C_{\mathrm{e}}$ was varied over four orders of magnitude. The main conclusion is that the explosion energy and the total mass of iron group elements (represented by ${ }^{56} \mathrm{Ni}$ ) is maximal for $C_{\mathrm{e}} \sim 10^{4}$. If the ignition process becomes even more rapid, bubbles of burning material are generated at such high rate that most of the bubbles merge before they can appreciably expand and deform due to the Rayleigh-Taylor instability. This effect tends to inhibit the growth of the flame surface area and, thus, reduces the explosion energy. More or less the same trend was observed for simultaneous ignition with varying bubble number by Röpke et al. (2005).

For $C_{\mathrm{e}} \ll 10^{3}$, there are only few ignitions per octant. In this case, the explosion is weak and the total mass of iron group elements is less than $0.5 M_{\odot}$. Setting $C_{\mathrm{e}}=10$, the white dwarf becomes barely unbound. Nevertheless, the ignition process 
Table 1. Parameter study with initial spatial resolution $\Delta=4.75 \mathrm{~km}$ in the uniform part of the numerical rid. The total spherical angle covered by the simulation domain is $\Omega$, the total number of ignitions per octant is $I_{\pi / 2}$, and the last ignition event occurs at time $t_{\text {last }}$.

\begin{tabular}{rllrccccc}
\hline \hline$C_{\mathrm{e}}$ & $R / \Lambda$ & $\Omega$ & $I_{\pi / 2}$ & $t_{\text {last }}[s]$ & $E_{\text {nuc }}\left[10^{51} \mathrm{erg}\right]$ & $E_{\text {kin }}\left[10^{51} \mathrm{erg}\right]$ & $M_{\mathrm{Ni}} / M_{\odot}$ & $M_{\mathrm{Mg}} / M_{\odot}$ \\
\hline $10^{1}$ & 0.5 & $\pi / 2$ & 2 & 0.200 & 0.516 & 0.008 & 0.242 & 0.165 \\
$10^{2}$ & 0.5 & $\pi / 2$ & 4 & 0.096 & 0.827 & 0.320 & 0.413 & 0.218 \\
$10^{3}$ & 0.5 & $\pi / 2$ & 30 & 0.508 & 1.139 & 0.631 & 0.629 & 0.187 \\
$5 \times 10^{3}$ & 0.5 & $\pi / 2$ & 65 & 0.177 & 1.219 & 0.711 & 0.690 & 0.170 \\
$10^{4}$ & 0.25 & $\pi / 2$ & 65 & 0.199 & 1.099 & 0.591 & 0.603 & 0.189 \\
$10^{4}$ & 0.5 & $\pi / 2$ & 115 & 0.323 & 1.261 & 0.753 & 0.707 & 0.188 \\
$10^{4}$ & 1.0 & $\pi / 2$ & 190 & 0.203 & 1.157 & 0.649 & 0.643 & 0.184 \\
$2 \times 10^{4}$ & 0.5 & $\pi / 2$ & 131 & 0.222 & 1.162 & 0.654 & 0.643 & 0.188 \\
$10^{5}$ & 0.5 & $\pi / 2$ & 284 & 0.306 & 1.131 & 0.623 & 0.629 & 0.177 \\
$10^{2}$ & 0.5 & $4 \pi$ & 6 & 0.703 & 0.985 & 0.478 & 0.523 & 0.201 \\
$5 \times 10^{3}$ & 0.5 & $4 \pi$ & 59 & 0.237 & 1.274 & 0.766 & 0.715 & 0.188 \\
$10^{5}$ & 0.5 & $4 \pi$ & 305 & 0.159 & 1.165 & 0.657 & 0.647 & 0.184 \\
\hline
\end{tabular}
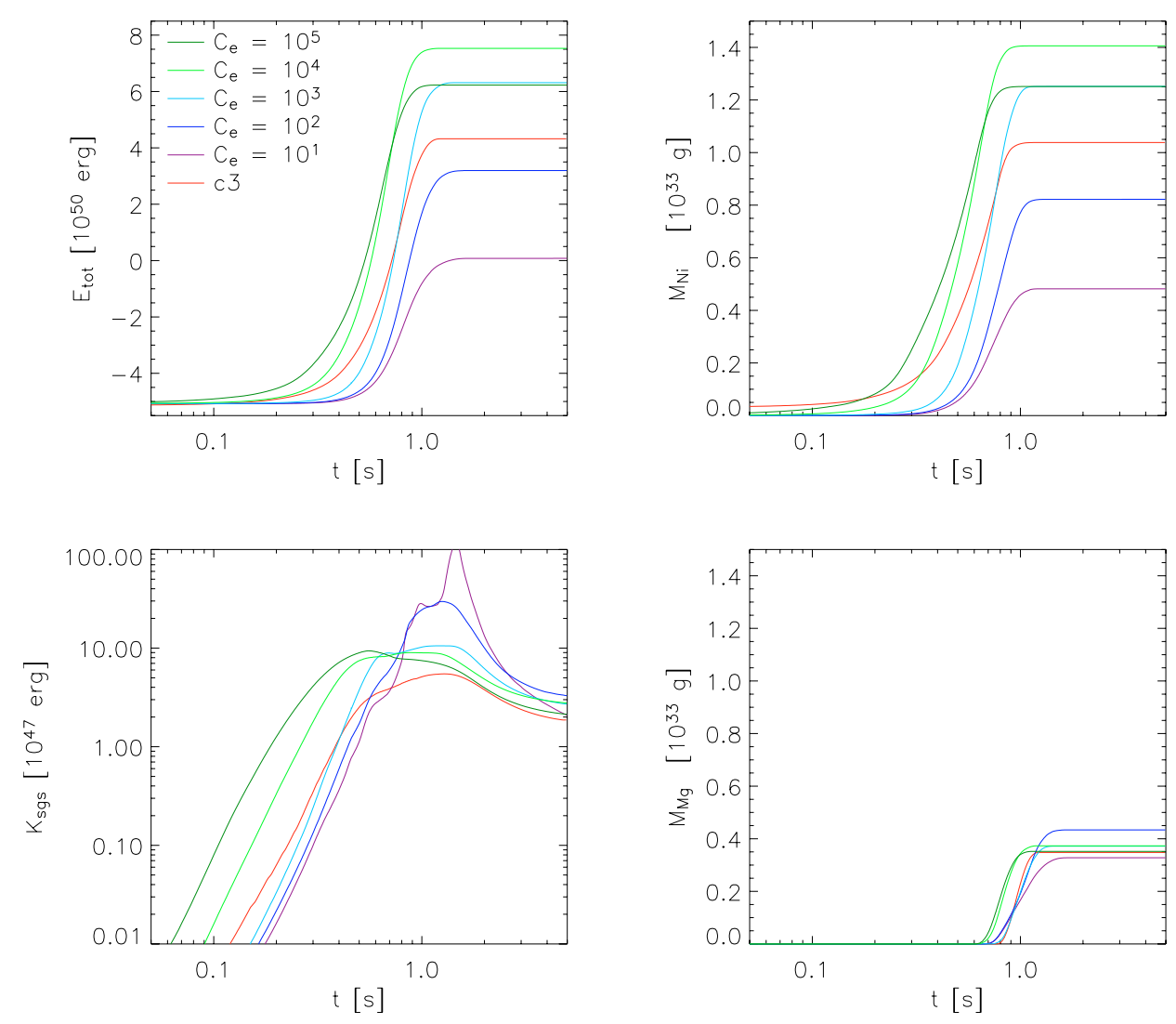

Fig. 1. Evolution of the total energy, the masses of iron group and intermediate mass elements, respectively, as well as the subgrid scale turbulence energy in simulations with axisymmetric initial flame (c3) and stochastic ignition with varying exponentiation parameter $C_{\mathrm{e}}$, respectively. The computational domain is a single octant with $N=128^{3}$ grid cells.

ceases because the probability of further ignitions becomes virtually zero once the star is expanding appreciably. This is simply a consequence of the power-law dependence of the factor $S_{\text {nuc }} / c_{\mathrm{P}}$ in formula (5) on the background temperature and density. In general, the ignition process terminates after a few tenths of a second regardless of the exponentiation parameter (see Table 1). The case $C_{\mathrm{e}}=1$ corresponds to roughly one ignition per second and would fail to produce an explosion at all. On the other hand, there appears to be a remarkable robustness for $C_{\mathrm{e}} \gtrsim 10^{3}$. The variation in the total nuclear energy release is less than $10 \%$ for all simulations with exponentiation parameter in excess of $10^{3}$, while the number of ignition events may change by a factor as large as ten. Changing the ratio of the mixing length to the thermal radius has more or less the same effect as varying the exponentiation parameter. If $R / \Lambda$ increases, the probability of ignition becomes higher. However, we found that choosing $R / \Lambda$ significantly smaller than 0.5 introduces an artificial cutoff in the range of ignition radii. For this reason, we set $R / \Lambda=0.5$.

The time evolution of the total energy is plotted for selected simulations in panel (a) of Fig. 1. One can clearly see the faster progression of the explosion with increasing number 
of ignitions and the reduced rate of growth for $C_{\mathrm{e}} \sim 10^{5}$. The final kinetic energy of $7.5 \times 10^{50} \mathrm{erg}$ in the case $C_{\mathrm{e}} \sim 10^{4}$ is about $75 \%$ larger than for the reference simulation with a perturbed axisymmetric initial flame (c3). The production of iron group and intermediate mass elements is shown in panels (b) and (d), respectively. It becomes clear that the statistical properties of the ignition process are crucial for the nucleosynthesis of iron group elements, whereas the the final amount of intermediate mass elements, which are represented by ${ }^{24} \mathrm{Mg}$, appears to be fairly robust. However, it is important to note that burning is terminated at the density threshold of $10^{7} \mathrm{~g} \mathrm{~cm}^{-3}$ because we still lack a sensible treatment of the distributed burning regime during the late stage of the explosion. Continuing the burning process into this regime might entail significant changes in the predictions of intermediate mass element production.

The integrated subgrid scale turbulence energy $K_{\mathrm{sgs}}$ is plotted in panel (c) of Fig. 1. In the early phase of the explosion, one can discern power-law growth of $K_{\mathrm{sgs}}$, where the exponent appears to be universal for stochastic ignition. There is merely a temporal shift of the graph depending on the exponentiation parameter. From $t \approx 0.5 \mathrm{~s}$ onwards, the increase of SGS turbulence energy diminishes. This marks the onset of the fully turbulent regime. The peak of $K_{\mathrm{sgs}}$ is increasingly delayed as the ignition process progresses slower for smaller values of $C_{\mathrm{e}}$. At still later time, the turbulence energy decreases due to the rapid bulk expansion and the quenching of the burning process which supplies energy to the flow. The exceptionally high peak values of $K_{\mathrm{sgs}}$ for the less energetic explosions are caused by a major fraction of the released energy stirring the white dwarf matter into turbulent motion rather than being consumed by bulk expansion. In this respect, the "dud explosions" obtained in the case of slow ingition are more like an overflowing boiling pot.

With regard to the question whether deflagration models can account for observed type Ia supernovae, it is crucial to look at the distribution of nuclear species at a time when the explosion ejecta enter the phase of homologous expansion. To that end we performed three full-star simulations and analysed the ejecta at $t=5.0 \mathrm{~s}$. From the statistics listed in Table 1 it might appear that the sensitivity to the ignition process is less pronounced than for the single-octant simulations, possibly due to the absence of artifical boundary conditions. However, Figs. 2-4 illustrate that there are, indeed, substantial differences in the final outcome. For each simulation, the stratification of the mass density is shown in two-dimensional sections through the centre of the computational domain. It is instructive to compare the partial densities of unburned carbon and oxygen, iron group elements ("Ni") and intermediate mass elements ("Mg"). In the case $C_{\mathrm{e}}=10^{2}$, the contour sections on the top of Fig. 2 show that a big lump of unburned material prevails in the central region. The corresponding fractional masses as function of radial velocity are plotted at the bottom of Fig. 2. Given the total amount of roughly $0.5 M_{\odot}$ of iron group elements, such an explosion definitely does not resemble any observed SN Ia. Increasing the exponentiation parameter, however, we obtain an ever more stratified distribution of nuclear species. For $C_{\mathrm{e}}=5 \times 10^{3}$, the nuclear ash appears to be mixed with unburned material over a wide range of radii and radial velocities, respectively (Fig. 3). In the case of most rapid
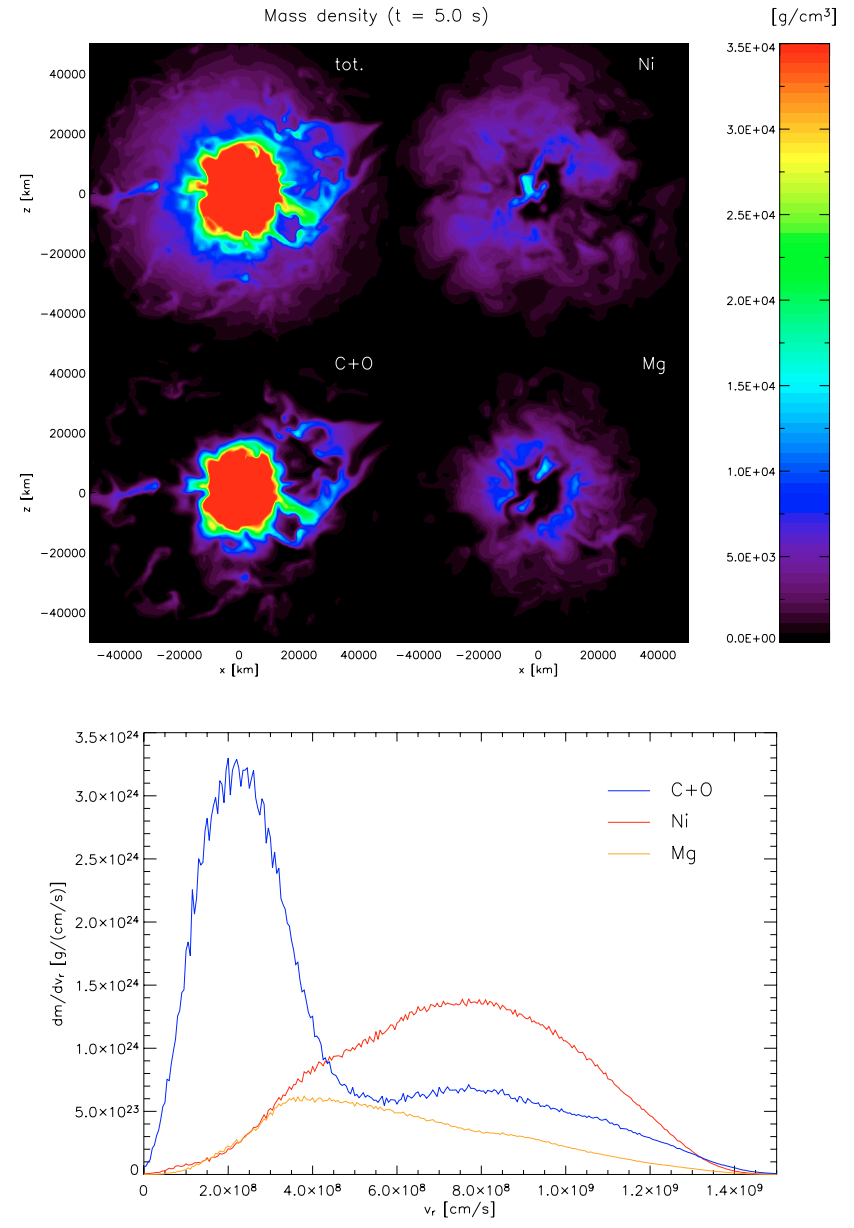

Fig. 2. Total and fractional mass densities in a central section and the corresponding probability density functions in radial velocity space for a full-star simulation with $C_{\mathrm{e}}=10^{2}$ at $t=5.0 \mathrm{~s}$.

ignition with $C_{\mathrm{e}}=10^{5}$, a clear trend towards concentration of nickel in the centre becomes apparent, while carbon and oxygen remain mostly in the outer layers. This is different from the results reported by Röpke et al. (2005) for simultaneous ignitions, where the material at small radial velocities appears to be depleted of carbon and oxygen for a total number of initially burning bubbles of the order one hundred, while further increasing the number of bubbles reverses the trend.

For a more detailed account of the flame evolution, we performed in addition one full-star simulation with the intermediate exponentiation parameter $C_{\mathrm{e}}=5 \times 10^{3}$ using $N=512^{3}$ numerical cells, i.e. twice the initial spatial resolution. The ignition process for this simulation is illustrated in Fig. 5. It might seem unexpected that relatively few ignitions occur close to the centre. However, this is a natural consequence of the scaling of the ignition probability with the volume. Moreover, most of the material near the centre is burned quickly and so there remains little space for further ignitions. The bulk of ignition events takes place at radii in the range between 50 and $250 \mathrm{~km}$ from the centre. The relatively large radial spread might be an artifact of the statistical rather than local evaluation of the temperature excess $\delta T_{\mathrm{b}}$ in expression (5) for the inverse time scale of the Poisson process. In consequence, we definitely see the requirement of an improved modelling of the temperature fluctuations. 

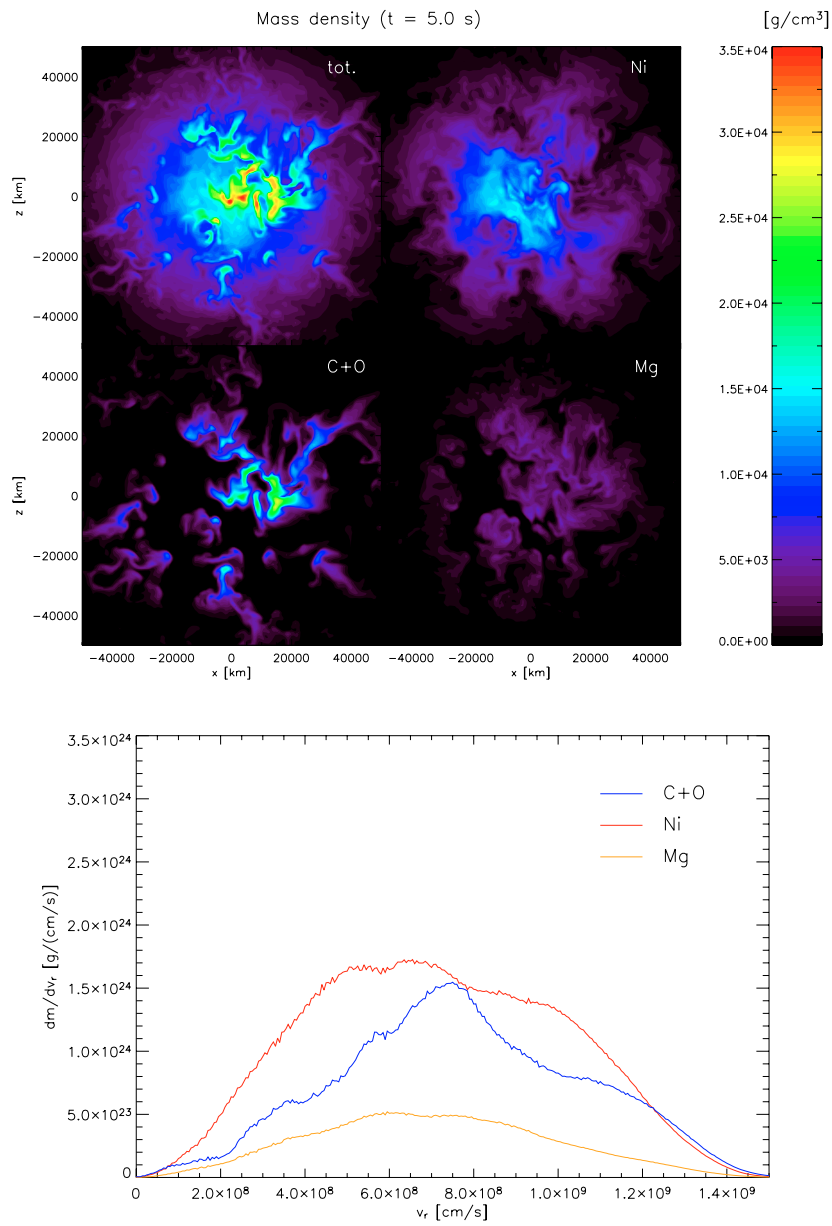

Fig. 3. Total and fractional mass densities in a central section and the corresponding probability density functions in radial velocity space for a full-star simulation with $C_{\mathrm{e}}=5 \times 10^{3}$ at $t=5.0 \mathrm{~s}$.

The conceptual design of a fluctuation model which allows for the computation of the spatiotemporal distribution has recently been proposed by Kerstein (2005).

Three-dimensional snapshots of the flame fronts in the simulation with high resolution are shown in Fig. 6. The subgrid scale turbulence velocity which determines the turbulent flame speed is indicated by contours at the flame surface. At time $t=0.1 \mathrm{~s}$ (a), one can see a large number of small bubbles generated by the stochastic ignition process. As the burning bubbles are floating outwards from the centre they begin to form the typical Rayleigh-Taylor mushroom shapes (b). At $t=0.5 \mathrm{~s}$, the flames mostly have merged into a single structure which is subject to intense turbulent flow (c). Apart from the small-scaling wrinkling of the flame front one can discern several dominating modes. Particularly, a pronounced asphericity emerges after most of the explosive burning has come to pass (d). The formation of dominating modes is a consequence of the fast growth of Rayleigh-Taylor instabilities seeded at large radii. Hence, the evolution of the flames is highly sensitive to the radial distribution of the ignitions. This implication is particularly interesting in the light of recently found observational evidence for asphericity of type Ia supernova ejecta (Leonard et al. 2005). The late-time evolution and, particularly, the distribution of nuclear species was found to be quite similar to the $256^{3}$ run.
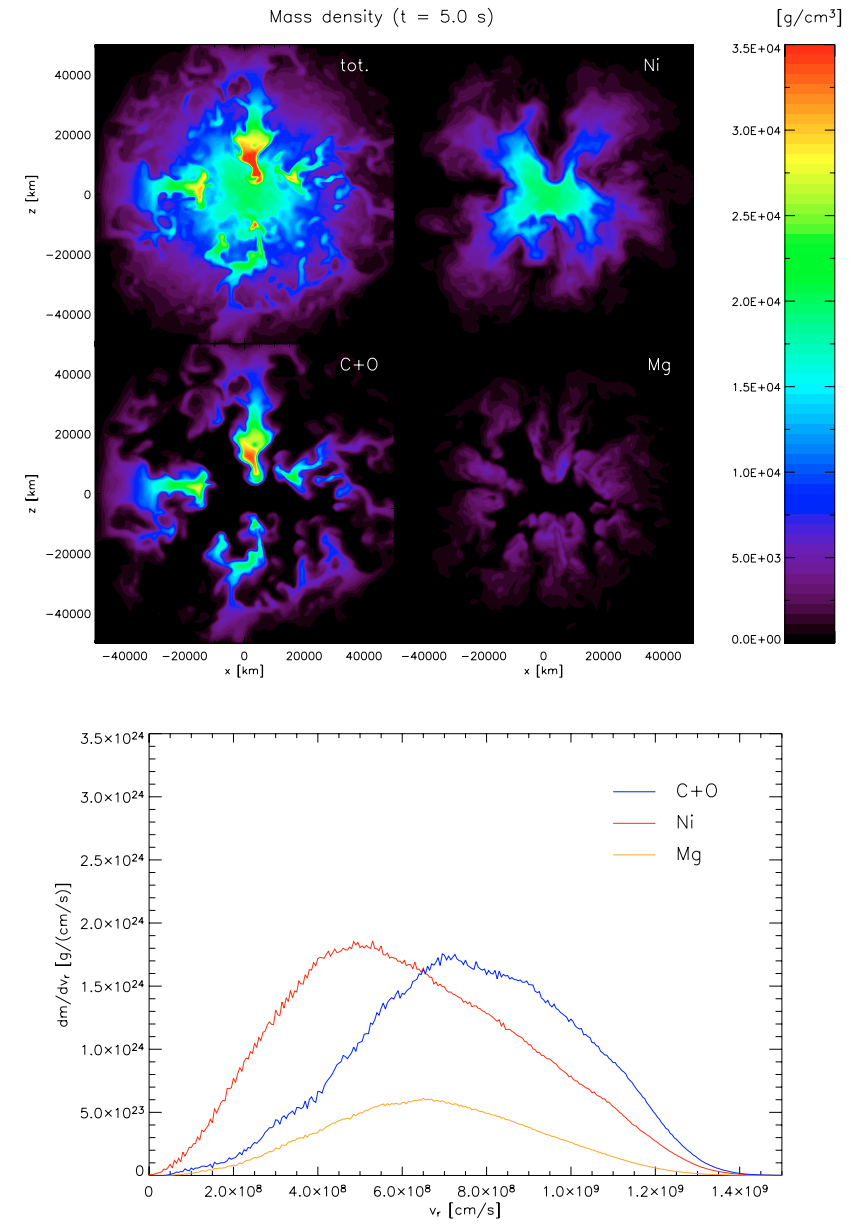

Fig. 4. Total and fractional mass densities in a central section and the corresponding probability density functions in radial velocity space for a full-star simulation with $C_{\mathrm{e}}=10^{5}$ at $t=5.0 \mathrm{~s}$.

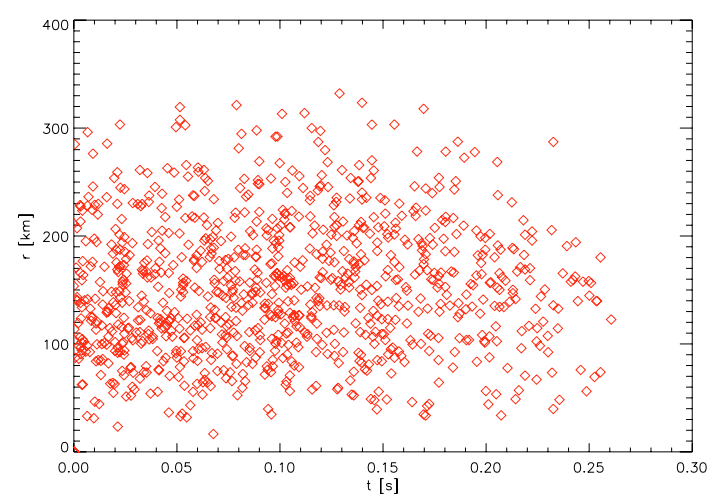

Fig. 5. Plot of the radii of ignition events as function of time in the full-star simulation with $N=512^{3}$.

\section{Conclusion}

We have achieved a first step toward the dynamical modelling of the ignition process in three-dimensional large-scale simulations of thermonuclear supernovae. There is one free parameter, called the exponentiation parameter $C_{\mathrm{e}}$, which controls the statistical properties of the ignition process, especially, the total number of ignition events. Varying $C_{\mathrm{e}}$ in the numerical simulations alters the total mass of iron group elements and the 


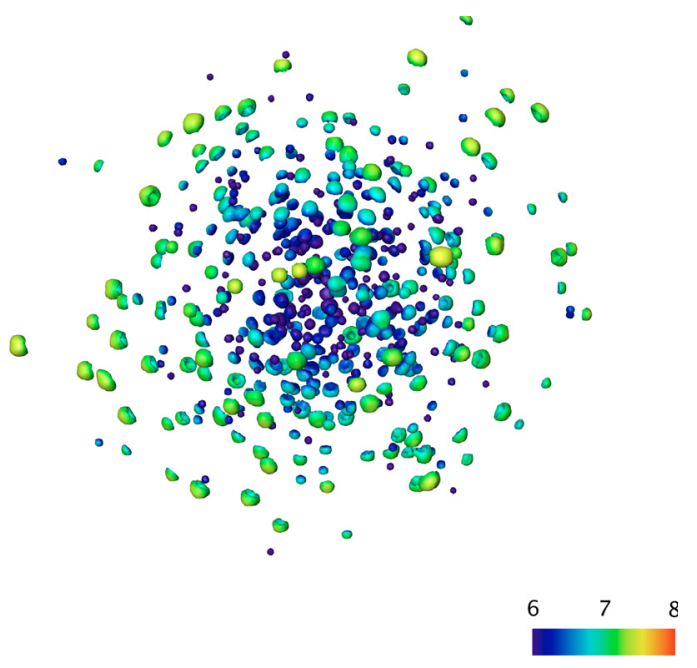

(a) $t=0.1 \mathrm{~s}, N_{\text {uni }}=320^{3}, X_{\text {uni }}=815 \mathrm{~km}$

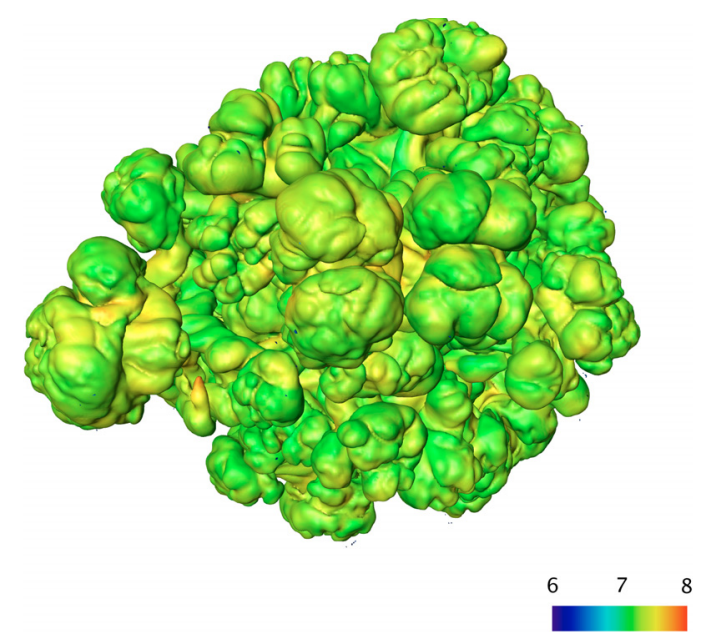

(c) $t=0.5 \mathrm{~s}, N_{\text {uni }}=396^{3}, X_{\text {uni }}=2339 \mathrm{~km}$

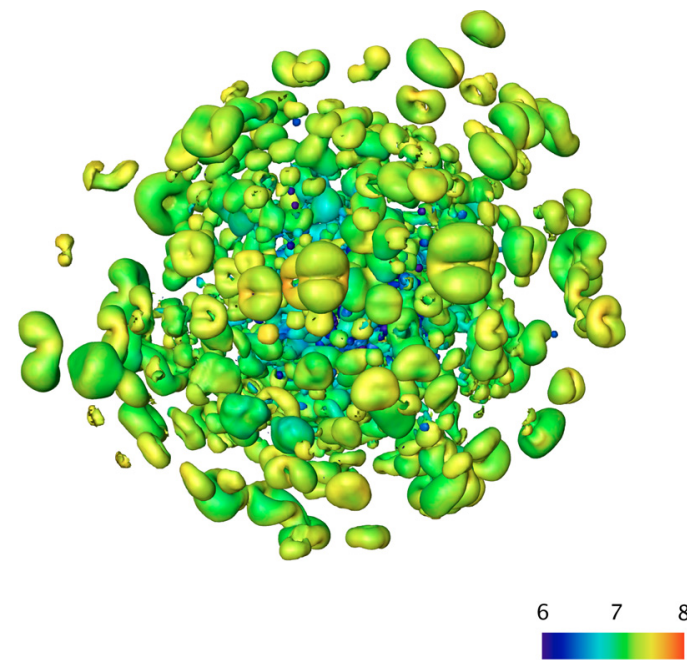

(b) $t=0.25 \mathrm{~s}, N_{\text {uni }}=340^{3}, X_{\text {uni }}=1125 \mathrm{~km}$

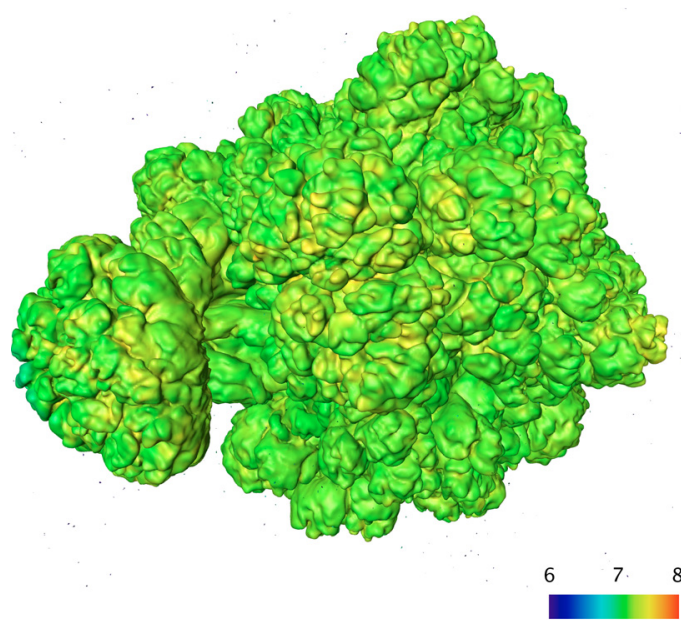

(d) $t=0.75 \mathrm{~s}, N_{\text {uni }}=438^{3}, X_{\text {uni }}=5177 \mathrm{~km}$

Fig. 6. Flame evolution in a full-star simulation with contours of the subgrid scale turbulence velocity in logarithmic scaling. For each snapshot, the physical time $t$ and the number of cells, $N_{\text {uni }}$, as well as the size $X_{\text {uni }}$ of the uniform part of the numerical grid which encloses the burning region are specified.

stratification of burning products. Particular ignition events can affect the morphology of the explosion ejecta as some modes grow faster than others. In fact, we found noticeable asphericity of the developing flame front even for high values of $C_{\mathrm{e}}$, where the statistical distribution of ignitions is nearly spherically symmetric.

If the total number of ignitions is less than one hundred in the whole core, only weak explosions with small total masses of iron group elements result. Although certain type Ia supernovae appear to produce, for instance, as little as $0.1 M_{\odot}$ of nickel (Stritzinger et al. 2005), the exceptionally large amount of left-over carbon and oxygen found in the simulations with small number of ignitions renders them unlikely to correspond to real events. The picture might change, however, if burning at low density is properly taken into account and, possibly, substantially larger amounts of intermediate mass elements are produced.
For a larger number of ignitions, on the other hand, strong explosions may result from pure deflagrations. However, even the most energetic explosions found in our simulations can not account for the upper range of observed nickel masses. In this respect, we arrive at the same conclusion as Röpke et al. (2005) for simultaneous multi-spot ignitions. On the other hand, we found that the explosion ejecta tend to exhibit an increasingly layered structure with growing number of ignition events. Consequently, the stochastic ignition model catches up with simulations in which a delayed detonation is initiated. Apart from that, Stritzinger et al. (2005) have found indications that possibly more nickel is mixed into the outer layers than previously assumed. This would confirm the prediction of simulations with an intermediate number of ignitions, say, about 50 per octant (Figs. 3 and 6). Nevertheless, if the possibility of more than a few ignitions occurring within a tenth of a second was excluded by advances in understanding the 
pre-supernova core evolution, then the deflagration model would be ruled out as an explanation for typical observed $\mathrm{SNe}$ Ia.

Acknowledgements. We thank Alan Kerstein and Stanford Woosley for helpful discussions and Friedrich Röpke for kindly sharing the comoving grid implementation. The simulations were run on the HLRB of the Leibniz Computing Centre in Munich. This work was supported by the Alfried Krupp Prize for Young University Teachers of the Alfried Krupp von Bohlen und Halbach Foundation.

\section{References}

Benetti, S., Cappellaro, E., Mazzali, P. A., et al. 2005, ApJ, 623, 1011 Gamezo, V. N., Khokhlov, A. M., \& Oran, E. S. 2004, Phys. Rev. Lett., 92,211102

Gamezo, V. N., Khokhlov, A. M., \& Oran, E. S. 2005, ApJ, 623, 337

García-Senz, D., \& Bravo, E. 2005, A\&A, 430, 585

Kerstein, A. 2005, private communication
Leonard, D. C., Li, W., Filippenko, A. V., Foley, R. J., \& Chornock, R. 2005, accepted for publication in ApJ, preprint [arXiv:astro-ph/0506470]

Livne, E., Asida, S. M., \& Hoeflich, P. 2005, ApJ, 632, 443

Niemeyer, J. C., \& Hillebrandt, W. 1995, ApJ, 452, 769

Press, W. H., Teukolsky, S. A., Vetterling, W. T., \& Metcalf, M. 1996, Numerical Recipes in Fortran (Cambridge University Press)

Röpke, F. K., \& Hillebrandt, W. 2005, A\&A, 431, 635

Röpke, F. K., Hillebrandt, W., Niemeyer, J. C., \& Woosley, S. E. 2005, A\&A, submitted, preprint [arXiv: astro-ph/0510474]

Schmidt, W., Hillebrandt, W., \& Niemeyer, J. C. 2005, Combust. Theory Modelling, 9(4), 693

Schmidt, W., Niemeyer, J. C., Hillebrandt, W., \& Röpke, F. K. 2005, A\&A, submitted

Stritzinger, M., Leibundgut, B., Walch, S., \& Contardo, G. 2005, A\&A, submitted, preprint [arXiv: astro-ph/0506415]

Woosley, S. E., Wunsch, S., \& Kuhlen, M. 2004, ApJ, 607, 921

Wunsch, S., \& Woosley, S. E. 2004, ApJ, 616, 1102 